\title{
Domain pattern formation and kinetics on ferroelectric surfaces under thermal cycling using scanning force microscopy
}

\author{
V. Likodimos, ${ }^{*}$ M. Labardi, and M. Allegrini \\ INFM and Dipartimento di Fisica, Università di Pisa, Via F. Buonarroti 2, I-56127 Pisa, Italy \\ (Received 15 May 2001; revised manuscript received 16 July 2001; published 1 July 2002)
}

\begin{abstract}
Scanning force microscopy in the dynamic contact mode with resonance enhanced domain contrast is applied to study in situ pattern formation and relaxation kinetics of ferroelectric domains on the cleavage surface of triglycine sulfate subjected to successive annealing-cooling cycles. Substantial differences in the domain morphology and kinetics are disclosed upon cooling at temperatures close to $T_{c}$. Convolution of stripe domains with residual domain nuclei and random pinning forces due to intrinsic defects are suggested to account for variations in the domain structure and slowing down of domain kinetics.
\end{abstract}

DOI: 10.1103/PhysRevB.66.024104

PACS number(s): 77.80.Dj, 68.37.Ef, 64.60.My

\section{INTRODUCTION}

Ferroelectric materials are currently the objective of intensive research investigations due to their potential integration in a wide range of technological applications, the most challenging being their use as nonvolatile memory elements, based on the high storage capacity of scanning probe techniques. ${ }^{1}$ High-resolution studies of the domain microstructure and relaxation kinetics have attracted particular interest due to the intimate relation of domain dynamics and the practical utilization of these materials depending essentially on the microengineering of ferroelectric domains and the suppression of drawbacks like retention loss. ${ }^{2}$

Scanning force microscopy (SFM) has offered unique opportunities for noninvasive imaging of the domain structure on ferroelectric surfaces with high spatial resolution, hardly accessible by conventional domain visualization methods. ${ }^{3}$ Among different variants, voltage-modulated SFM, ${ }^{4}$ relying on the electrical-electromechanical response to an external ac voltage, ${ }^{5}$ has emerged as a powerful technique to study polarization domains and their dynamics in ferroelectric materials. ${ }^{1,2,6-17}$ Due to the finite vibration amplitude of the cantilever in contact conditions, the corresponding operation mode has been termed as dynamic contact electrostatic force microscopy (DC-EFM), ${ }^{9,12,14,18}$ an abbreviation adopted hereafter to discriminate it from the noncontact EFM mode. An important advantage of this method is the suppression of image artifacts due to the convolution of electric with topographic properties, inherent in other SFM imaging modes. ${ }^{18}$ Sensitivity in DC-EFM can be significantly increased using the marked domain contrast enhancement recently revealed by the excitation of cantilever resonance modes in contact conditions. ${ }^{18}$

The potential of DC-EFM has been exploited to probe in situ the paraelectric-ferroelectric phase transition in triglycine sulfate (TGS) ferroelectric crystals by the implementation of variable temperature measurements. ${ }^{11-13,16}$ The temperature dependence of the domain density and its reversibility close to the Curie temperature $T_{c}$ have been measured, ${ }^{12}$ allowing comparison with mean-field predictions established by macroscopic measurements. ${ }^{19,20}$ Measurements of the temporal domain evolution after quenching from above $T_{c}$ have shown size-dependent domain relaxation rates, while the interaction between adjacent small domains of the same polarity has been implicated to account for the nonmonotonic domain size variation. ${ }^{9}$ However, diverse domain configurations have been observed on TGS cleavage surfaces subjected to different thermal treatments. ${ }^{11,13}$ Systematic utilization of the resonance enhancement method in DC-EFM allowed the observation of a nonlinear temporal variation of the correlation length and the presence of dynamic scaling in the growth morphology of TGS domain kinetics monitored in real time after quenching into the ferroelectric phase. ${ }^{14}$ Experimental evidence for the crossover to the slow logarithmic growth law predicted for random-bond pinning forces has been thus derived. ${ }^{14}$ Further experiments on TGS crystals quenched at different temperatures below $T_{c}$ have shown the presence of thermally activated domain growth, consistent with quenched disorder due to randombond defects, which was earlier proposed, yet not experimentally verified, for ferroelectric materials. ${ }^{15}$

In this work, voltage-modulated SFM in the dynamic contact mode is applied to investigate in situ the transformation and relaxation kinetics of the domain structure on the cleavage surface of TGS crystals under successive annealingquenching thermal cycles through the ferroelectric phase transition. Quantitative image analysis, based on the resonance enhanced domain contrast of DC-EFM, is exploited to disclose microscopic information of the morphology, refinement, and growth of ferroelectric domains at different stages of thermal treatment. Intrinsic defects and the buildup of random pinning forces are inferred to be a major factor in pattern formation and most importantly domain kinetics.

\section{EXPERIMENT}

Voltage-modulated SFM measurements were performed in the dynamic contact mode ${ }^{18}$ on TGS single crystals undergoing a second-order phase transition to the ferroelectric phase at $T_{c} \approx 322 \mathrm{~K} \cdot{ }^{19,20}$ Slices (thickness $\approx 0.7 \mathrm{~mm}$ ) were cleaved in air along the (010) plane that comprises the cross section of domains with spontaneous polarization $P_{s}$ perpendicular to the inspected surface. Conductive $n^{+}$-silicon cantilevers (Nanosensors) of contact mode type ( $k$ $\approx 0.1 \mathrm{~N} / \mathrm{m}, f_{\text {res }} \approx 13 \mathrm{kHz}$ ) were used. It should be noted 

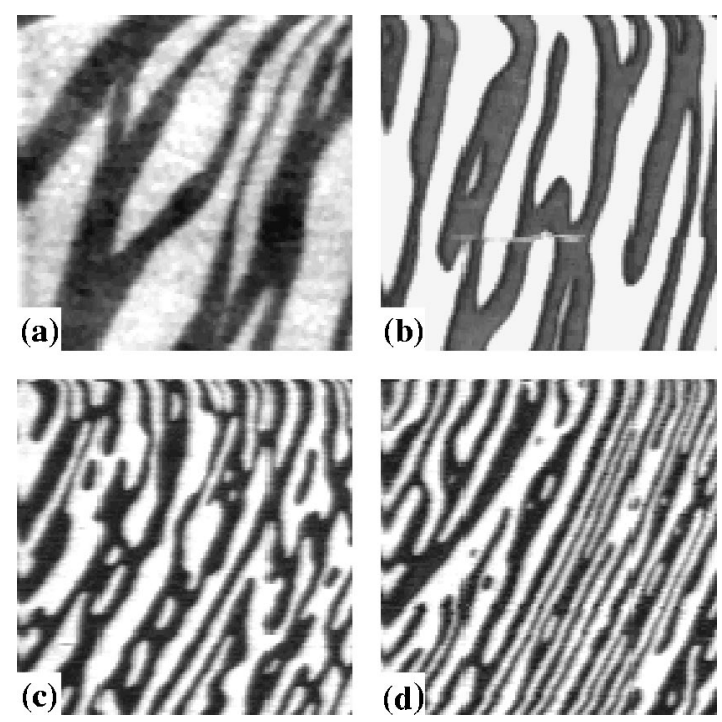

FIG. 1. Domain images of the (010) polar surface of TGS recorded in the ferroelectric phase at $T_{1}=320 \mathrm{~K}$ immediately after the (a) first, (b) second, (c) third, and (d) fourth successive annealings at $T_{\mathrm{an}}=333 \mathrm{~K}$. The duration of the annealing is $125,110,110$, and $130 \mathrm{~min}$, respectively. The time interval between crossing $T_{c}$ and image acqusition is $\Delta t \approx 4 \mathrm{~min}$ in all cases. Scan size 20 $\times 20 \mu \mathrm{m}^{2}$.

that as the electric field near the tip apex is greatly enhanced due to its geometrical asymmetry, ${ }^{10}$ it could exceed the coercive field for TGS, which is of the order $10^{4}-10^{5} \mathrm{~V} / \mathrm{m}$ for static or low-frequency (e.g., $50 \mathrm{~Hz}$ ) fields. ${ }^{19}$ This effect was explored by measurements on TGS specimens with variable amplitude of the ac voltage, which showed no distortion of domain imaging in the voltage range of $1-10 \mathrm{~V}$, most likely due to the high frequency of the sustained cantilever vibration that effectively increases the coercive field. This result agrees with previous DC-EFM measurements employing amplitude values in the same voltage range $(1-10 \mathrm{~V})$ for noninvasive domain imaging of the TGS polar surface. ${ }^{7,9,12}$ Moreover, scanning a small window of the sample surface and subsequently zooming out showed no perturbation at the edges of the smaller scanned area, implying negligible influence of the probe field on the domain structure. The amplitude of the ac voltage was accordingly chosen to be $5 \mathrm{~V}_{\mathrm{rms}}$.

Resonance enhancement of domain contrast is attained by the pronounced amplification effect at the first flexural resonant mode of the contact-mode-type cantilever, which shifts to $64 \mathrm{kHz}$ for the present measurements. ${ }^{18,21}$ A dual lock-in amplifier was used to record the enhanced amplitude $(R)$ signal, where strong contrast is detected between oppositely polarized domains, apart from that of domain walls, through a sustained contact potential difference. ${ }^{21}$ In situ thermal annealing above $T_{c}$ and cooling at different temperatures into the ferroelectric phase were successively performed using a temperature-controlled stage $(\Delta T \leqslant 0.1 \mathrm{~K})$ mounted on the microscope head.

\section{RESULTS AND DISCUSSION}

Figure 1 shows domain $(R)$ images of a freshly cleaved
TGS specimen cooled in the ferroelectric phase at $T_{1}$ $=320 \mathrm{~K}$ after four successive thermal annealing treatments. Prior to each measurement the sample was annealed in air at a constant temperature $T_{\mathrm{an}}=333 \mathrm{~K}$ in the paraelectric phase and then rapidly cooled at a constant rate of $4 \mathrm{~K} / \mathrm{min}$ below $T_{c}$ at $T_{1}$. The annealing time was $125,110,110$, and 130 min, respectively. After each thermal treatment, domain relaxation was monitored for a few hours and then the annealling-cooling process was repeated. In all cases, domain images were recorded near the same surface region, while the time elapsed after crossing $T_{c}$ and image acquisition is approximately $4 \mathrm{~min}$. Immediately after the first annealing, a relatively coarse structure comprising extended lenticular domains is observed [Fig. 1(a)]. Domain walls are elongated nearly perpendicular to the $c$ axis, in accordance with the anisotropy of the TGS wall energy. ${ }^{22}$ A subsequent annealing-cooling treatment leads to a progressive refinement of the domain patterns that maintain their preferable orientation [Figs. 1(b)-1(d)]. A similar effect has been detected on TGS upon thermal annealing by scanning electron microscopy, where a minimum domain size of $2 \mu \mathrm{m}$ was resolved. $^{23}$ In that case, a decrease of the average domain width and evolution of lenticular to striation-type domains were detected at room temperature under three successive thermal treatments with increasing annealing time $(30,300$, $3000 \mathrm{~min})$. In this respect, it appears that the refinement of the domain structure after the almost isochronal, successive annealing at constant $T$ might be equivalent to an effective increase of the annealing time. The topographic images do not show any change of surface morphology after each thermal treatment. Such morphologic changes may take place at the unit cell scale that is hardly observable by SFM operating in air.

However, a marked distortion of the domain morphology is observed after the third and fourth annealing treatments. The accuracy of the temperature measurement $(\Delta T$ $\approx 0.1 \mathrm{~K})$ is identical for all domain images recorded after each annealing, excluding to a great extent its possible effect on the variation of the domain morphology. The corresponding domain structure reveals a high deformation of the domain walls [Fig. 1(c)] and subsequently transforms into a complicated pattern of fine stripe domains with many wedge fronts along with curved interfaces and small nuclei [Fig. 1(d)]. Comparison of these patterns with typical TGS domain configurations comprising either the irregular and lamellar patterns $^{22-24}$ or the complex domain structure resolved just below $T_{c}$ with the electron microscope decoration technique $^{25}$ reveals distinct differences. On the other hand, a close similarity can be evinced with the amorphous and strongly disturbed stripe domain patterns observed in quenched $\mathrm{NaNO}_{2}$ platelets. ${ }^{26}$ Such deformed structures have been shown to form from the initial stripe domain pattern through the nucleation and growth of antistripples, the characteristic nucleus of the incommensurate phase. Prompted by these observations, the temporal evolution of these domain configurations was pursued with constant recording conditions for approximately $300 \mathrm{~min}$.

Figures 2 and 3 show examples of the domain $(R)$ images recorded successively at $320 \mathrm{~K}$, after the third and fourth 


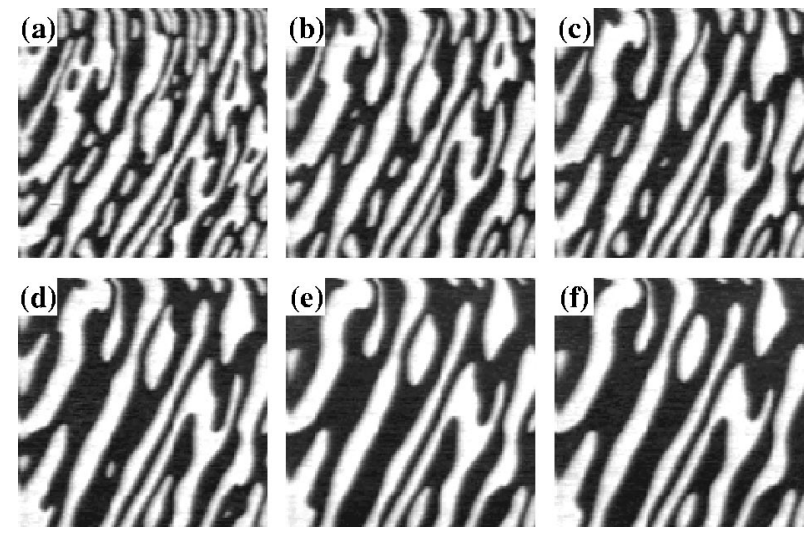

FIG. 2. Domain pattern ordering on the TGS (010) surface at $T_{1}=320 \mathrm{~K}$ after the third annealing treatment, shown in Fig. 1(c). The images have been successively recorded (a) $4 \mathrm{~min}$, (b) $18 \mathrm{~min}$, (c) $48 \mathrm{~min}$, (d) $110 \mathrm{~min}$, (e) $200 \mathrm{~min}$, and (f) $295 \mathrm{~min}$ after crossing $T_{c}$. Scan size $20 \times 20 \mu \mathrm{m}^{2}$.

annealing treatments, respectively. In the former case, small isolated domain nuclei gradually shrink until they disappear, while the highly curved interfaces become progressively smoother, implying a curvature driven growth mechanism (Fig. 2). In the latter case, minute domains vanish within a few minutes, while some of the finest stripes contract along their long axis, giving rise to stepped boundaries (depicted by arrows) in the initial growth stage [Figs. 3(a)-3(d)]. However, in the late-time regime, considerable retardation of the growth process is observed [Figs. 3(d)-3(f)]. The motion of the stepped domain interfaces is significantly hindered in the corresponding time scale, complying with the immobile steplike domains forming in quenched $\mathrm{NaNO}_{2}$ through the backward motion of adjacent wedge fronts. ${ }^{27}$

To quantify relaxation kinetics, the spatially averaged equal-time correlation function $C(r, t) \equiv\langle S(r, t) S(0, t)\rangle$, where $S(r, t)$ corresponds to the scalar order parameter field assuming the values +1 and -1 for $r$ residing in each of the
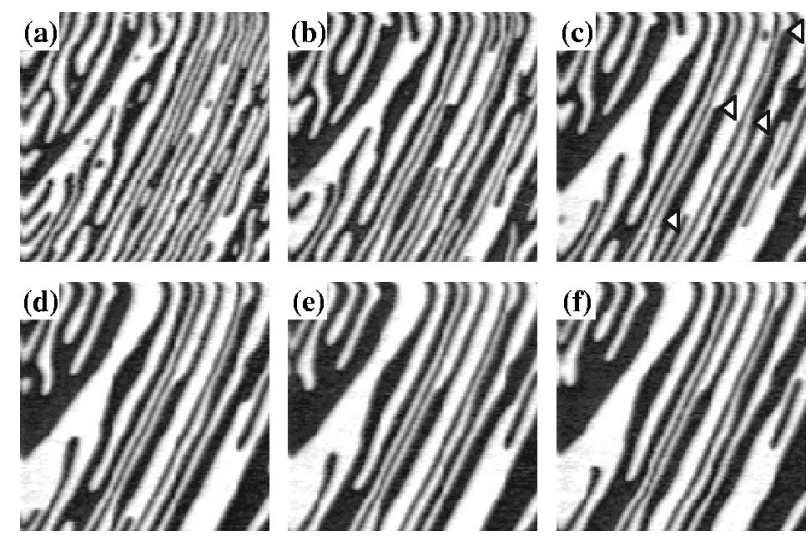

FIG. 3. Domain pattern ordering on the TGS (010) surface at $T_{1}=320 \mathrm{~K}$ after the fourth annealing, shown in Fig. 1(d). The images have been recorded (a) $4 \mathrm{~min}$, (b) $19 \mathrm{~min}$, (c) $48 \mathrm{~min}$, (d) 104 min, (e) $198 \mathrm{~min}$, and (f) $294 \mathrm{~min}$ after crossing $T_{c}$. Arrows in (c) depict the formation of steplike domain boundaries. Scan size 20 $\times 20 \mu \mathrm{m}^{2}$.

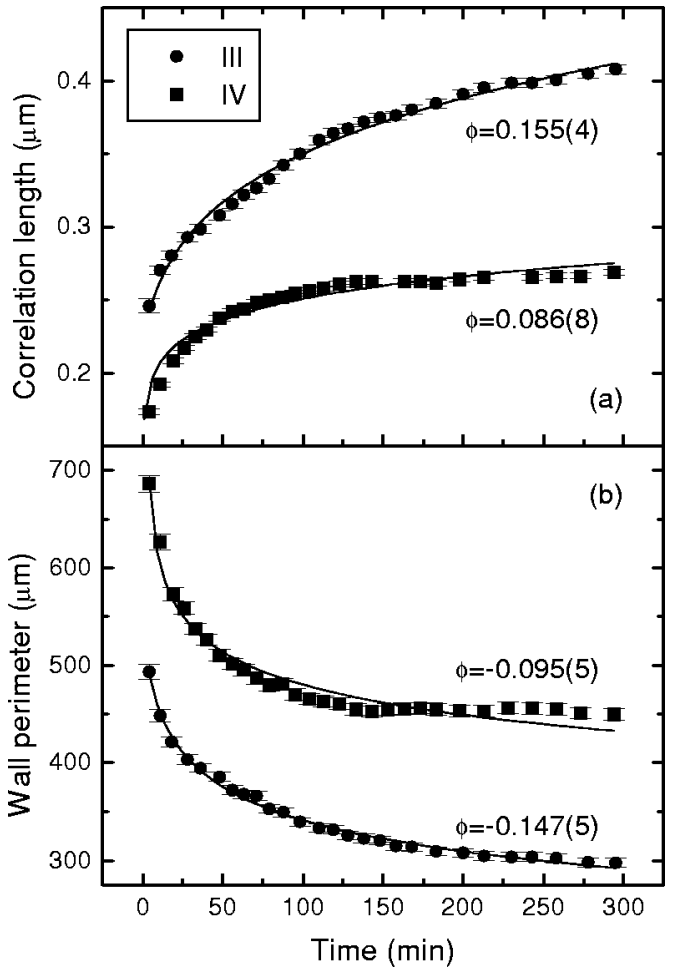

FIG. 4. Time dependence of (a) the correlation length $L(t)$ and (b) total domain perimeter $P(t)$ at $T_{1}=320 \mathrm{~K}$ after the third (III) and fourth (IV) annealing treatments. Solid lines correspond to the best-fit curves to the power law $\propto\left(t-t_{0}\right) \phi$ with growth exponents $\phi$ indicated for each data series and time offset values $t_{0}$ reported in the text.

two oppositely polarized states, ${ }^{28,29}$ were calculated along the horizontal direction of the domain patterns, applying the same image processing as before. ${ }^{11}$ The time dependence of the average domain size was then extracted using the characteristic correlation length $L(t)$, defined as the distance where $C(r, t)$ drops to half of its value at $r=0$, i.e., $C[r$ $=L(t), t]=1 / 2$, for a given time $t$. The resulting growth law for both annealing treatments is plotted in linear scales in Fig. 4(a). The nonlinear $L(t)$ variation can be fitted to the power law $L(t) \propto\left(t-t_{0}\right)^{\phi}$ where $t_{0}$ is a constant offset time and $\phi$ is the growth exponent characterizing the rate of domain coarsening, which for the purely curvature-driven domain growth of nonconserved systems is equal to $1 / 2$ and $1 / 3$ for the slower dynamics of conserved systems. ${ }^{28}$ The best-fit values obtained for the two successive annealings are $t_{0}=-5(3) \mathrm{min}, \phi=0.155(4)$, and $t_{0}=1(1) \mathrm{min}, \phi$ $=0.086(8)$ [Fig. 4(a)]. In both cases, the resulting growth exponents attain values considerably smaller than $1 / 3$, indicative of a much slower kinetics, in contrast with previous microscopic studies of TGS domain growth on the submillimeter, ${ }^{30,31}$ and recently submicron scales. ${ }^{14,15}$ Moreover, the kinetics is significantly slowed down after the last annealing, in accordance with the retardation of domain growth inspected on the corresponding patterns (Fig. 3). In this case, the simple power-law description breaks and crossover to a much slower temporal regime can be inferred at $t$ $>130 \mathrm{~min}$. This behavior is further corroborated by the time 


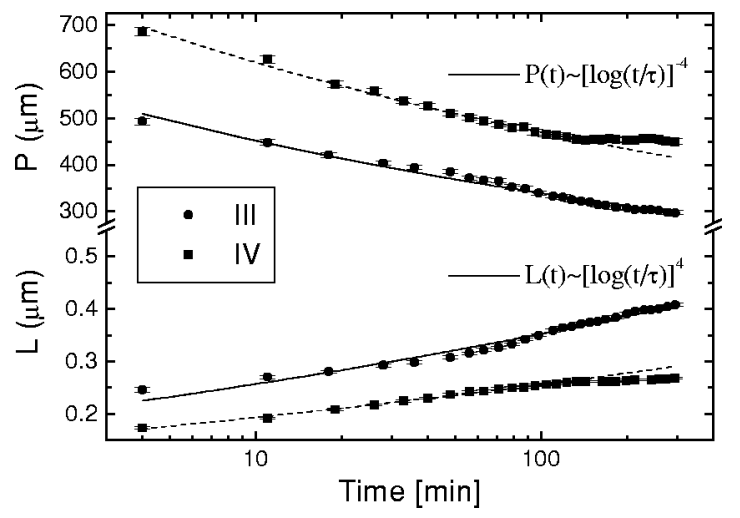

FIG. 5. Semilogarithmic plot of the correlation length $L(t)$ and total domain perimeter $P(t)$ at $T_{1}=320 \mathrm{~K}$ after the third (III) and fourth (IV) annealing treatments and the best-fit curves to the logarithmic growth law $L(t) \propto[\ln (t / \tau)]^{1 / \psi}$ with $|\psi|=1 / 4$. Crossover to a slower growth regime is seen by the deviation of the data points in IV from the dashed line derived from fitting in the earlier ordering stage.

dependence of the total domain perimeter $P(t)$ - that is, the total wall length-providing an independent measurement of the average domain size for both image series [Fig. 4(b)]. Fitting of the $P(t)$ data to the previous power law results in the values $t_{0}=-6(3) \mathrm{min}, \phi=-0.152(2)$ and $t_{0}$ $=2$ (1) $\min , \phi=-0.095(5)$ for the two thermal treatments, in good agreement with those derived from the $L(t)$ variation.

A plausible explanation of the reduced growth rate may be suggested considering the presence of quenched disorder due to randomly distributed defects ${ }^{14,15}$ and the consequent pinning effect on the domain wall motion. ${ }^{32-34}$ In that case, it was found that the logarithmic growth law $L(t)$ $\propto[\ln (t / \tau)]^{1 / \psi}$, with $\tau$ being a microscopic time scale and $\psi$ $=1 / 4$ pertinent for random-bond pinning forces in two dimensions, ${ }^{35,36}$ describes consistently the thermally activated domain growth detected on TGS quenched at different temperatures below $T_{c} \cdot{ }^{15}$ Figure 5 shows $L(t)$ and $P(t)$ in semilogarithmic scales, along with the fitting curves to the logarithmic time dependence. Although improved fits are obtained for the exponent value of $\psi=1 / 4$ rather than $\psi=1$ predicted for random-field quenched disorder, ${ }^{37}$ deviations from a single growth law are found in both cases. A small kink can be reliably discriminated at $t \approx 70 \mathrm{~min}$ in both $L(t)$ and $P(t)$ plots after the third annealing, while a clear crossover to a much slower ordering regime is found after the fourth annealing at $t \geqslant 130 \mathrm{~min}$. Similar behavior has been recently deduced, though less pronounced, in TGS domain kinetics at nearly the same time interval. ${ }^{14}$ To explore the latter behavior and the temporal evolution of the growth morphology, we examine the scaling properties of the correlation functions. Figure 6 shows plots of $C(r, t)$ as a function of the scaled distance $x \equiv r / L(t)$ for both data sets and all different times. All plots collapse onto a single curve up to a scaled distance $x \approx 2$ and then begin to disperse. The most appreciable deviation occurs for the last image series at $x$ $>2$ as time elapses [Fig. 6(b)]. The dynamic scaling form $C(r, t)=f[x \equiv r / L(t)]$ of the equal-time correlation function

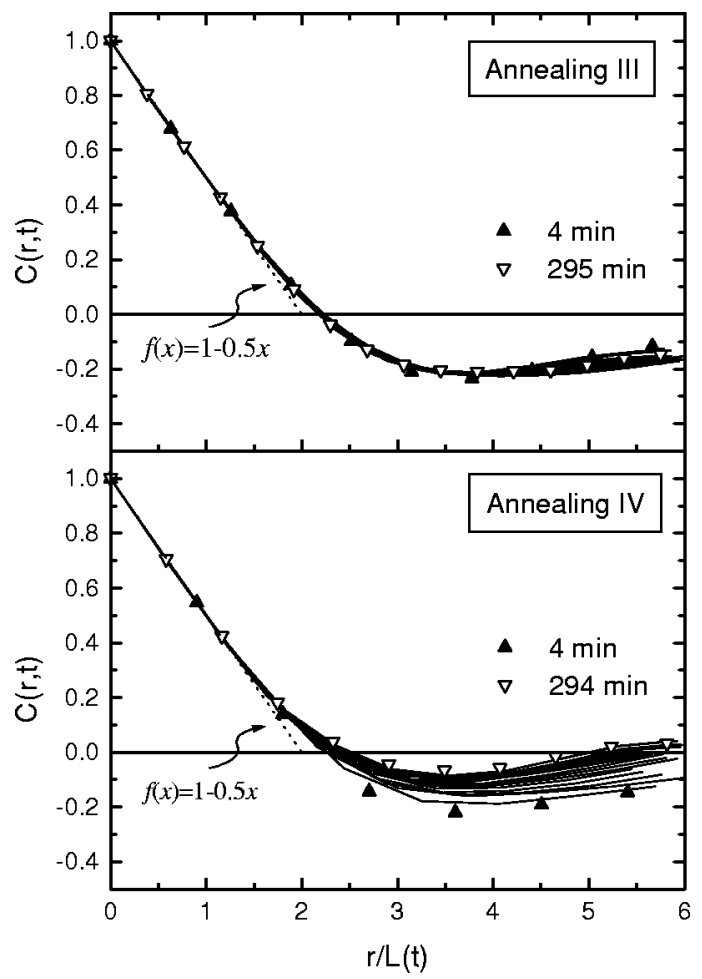

FIG. 6. Scaled correlation functions $C(r, t)=f[r / L(t)]$ of the TGS domain patterns for all different times after the third (III) and fourth (IV) annealings. Solid and open symbols show the correlation functions derived at the earliest and latest times. Dotted lines correspond to the linear decrement of the short-distance part of the scaling function according to $f(x)=1-0.5 x$.

can be thus claimed, implying the existence of a single length scale in the ordering process and the self-similar morphology of domain growth. The short-distance part of the scaled $C(r, t)$ decreases almost linearly with $x$ with a value of the linear coefficient of $f(x)$ close to the value of $\approx 0.5$ calculated for the two-dimensional Ising model, ${ }^{38}$ complying with theoretical predictions that the scaling function in random media is independent of the strength of randomness and identical to that of the pure systems. ${ }^{39-41}$

Domain pattern formation was subsequently investigated on the same specimen subjected to successive thermal cycles at lower temperature - that is, annealing at $T_{\text {an }}=333 \mathrm{~K}$ for $60 \mathrm{~min}$ followed by rapid cooling $(4 \mathrm{~K} / \mathrm{min})$ in the ferroelectric phase at $T_{2}=314 \mathrm{~K}\left(T_{2}<T_{1}<T_{c}\right)$. Figure 7 shows the domain $(R)$ images detected at $T_{2}$ after each thermal treatment, approximately on the same surface region with identical recording conditions as in the previous measurements. The origin of time is set at the time of crossing $T_{c}$ - that is, now 5-6 min. A drastic change of the domain morphology is observed (Fig. 7) in comparison with the domain patterns forming at higher temperature $T_{1}$ (Fig. 1). The domain structure now comprises long stripes decorated with smaller lenticular nuclei, similar to that recently observed by DC-EFM on TGS after quenching from above $T_{c}$ to room temperature. ${ }^{9}$ Successive annealing causes progressive refinement of the domain patterns by the nucleation of narrower stripes and smaller domain nuclei, which in several 


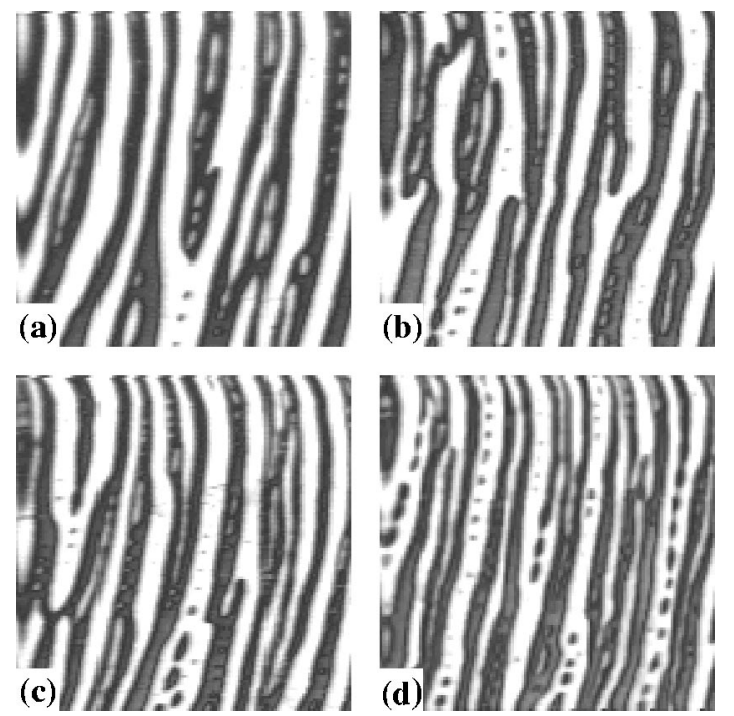

FIG. 7. Domain images of the TGS (010) polar surface recorded at $T_{2}=314 \mathrm{~K}$ immediately after the (a) first, (b) second, (c) third, and (d) fourth successive annealings at $T_{\text {an }}=333 \mathrm{~K}$. The duration of the annealing is $60 \mathrm{~min}$ and the time interval between crossing $T_{c}$ and image acquisition is $\Delta t \approx 5-6 \mathrm{~min}$ in all cases. Scan size 20 $\times 20 \mu \mathrm{m}^{2}$.

cases seem to arise from the "dissolution" of the finer domain stripes (Fig. 7). This type of domain configuration complies with the ordered arrangement of domain nuclei in rows along the [102] direction on the TGS polar surface in switching experiments, ${ }^{42}$ though with much lower density $\left(10^{-1} \mu \mathrm{m}^{-1}\right)$ than the present one.

Figure 8 shows examples of the temporal evolution of the TGS domain structure, measured after the last thermal treatment at $T_{2}$. Domain kinetics proceeds mainly by the rapid
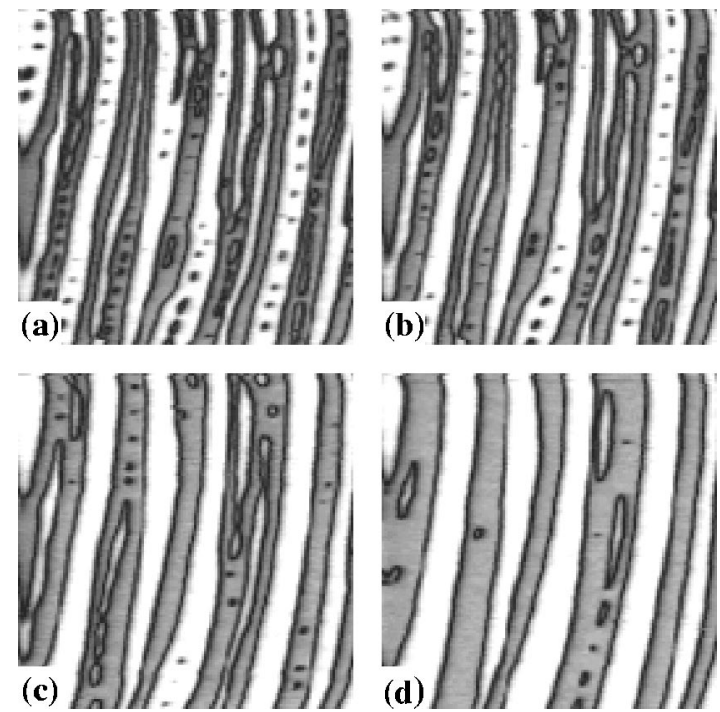

FIG. 8. Domain kinetics on the TGS (010) surface at $T_{2}$ $=314 \mathrm{~K}$ after the last annealing treatment shown in Fig. 7(d). The images have been successively recorded (a) $11 \mathrm{~min}$, (b) $18 \mathrm{~min}$, (c) $42 \mathrm{~min}$, and (d) $111 \mathrm{~min}$, after crossing $T_{c}$. Scan size 20 $\times 20 \mu \mathrm{m}^{2}$. shrinkage of the smaller nuclei and breaking up of fine domain stripes into new lenticular domains of smaller dimensions, which also contract rapidly until they disappear. A coarser stripe pattern is eventually formed [Fig. 8(d)], in a similar way as the inverse refinement process of the domain structure upon the successive annealing treatments. The coarsening process bears close resemblance to the piezoelectric domain clamping mechanism, ${ }^{43}$ which dominates the last stages of polarization reversal kinetics in TGS at moderate electric fields and larger spatial scale. ${ }^{44}$ This process appears to be energetically favorable regarding the elastic energy for a pattern of closely spaced domain stripes, when the width of the domains becomes comparable with their separation. However, the most interesting feature revealed by the temporal domain evolution is the presence of faster kinetics at $T_{2}$ compared with that at $T_{1}>T_{2}$ (Figs. 2 and 3). Apart from the different domain morphology at these temperatures, this behavior is in contrast with the slower kinetics that would be anticipated at $T_{2}$ in terms of thermally activated domain growth. ${ }^{15}$

A possible reason for this discrepancy may be suggested assuming that the peculiar domain configurations at $T_{1}$ [Figs. $1(c)$ and $1(d)$ ] originate from the convolution of stripe domains with isolated cigar-shaped domain nuclei emerging up to the crystal surface with elliptical cross sections. Residual domain nuclei have been identified in TGS crystals, due to the nonuniform defect structure giving rise to regions with essentially weakened dipole-dipole correlations. ${ }^{45}$ Their density on the TGS polar surface has been found to exhibit an anomalous rise with increasing electric field and temperature at $T>312 \mathrm{~K}$, and to be fairly stable upon brief annealing above $T_{c}$ or even prolonged repolarization in strong electric fields. ${ }^{45}$ The nucleation and growth of residual domains on the polar surface can be further assisted by the temperature gradient along the polar $b$ axis of the investigated specimen, whose free surface is in contact with the ambient environment. The temperature gradient and the accompanying polarization gradient increase considerably as temperature approaches $T_{c}$. Consequently, when cooling is rapidly performed at temperatures close to $T_{c}$, isolated domain nuclei may grow up to the polar surface at a high rate. Convolution of these domain nuclei characterized by slow growth velocities with the fine domain lamellae might then explain the anomalous curvature (Fig. 2) and stepped domain boundaries (Fig. 3) of the domain patterns at $T_{1}$, which, nevertheless, preserve their self-similar growth morphology in the subsequent coarsening process. Such a domain configuration may effectively reconcile the similarity with pattern formation in quenched $\mathrm{NaNO}_{2}$, where strongly deformed patterns have been observed to arise from the lamellar domain structure when the nucleation rate of antistripples is high and the growth velocity is low. ${ }^{26}$

\section{CONCLUSIONS}

Resonance enhanced DC-EFM is exploited to study in situ pattern formation and relaxation kinetics of ferroelectric 
domains on the cleavage surface of TGS crystals subjected to thermal cycles. Progressive refinement of the domain structure is systematically observed after each thermal treatment. Significant deformation of the domain structure is revealed close to $T_{c}$. Convolution of residual domain nuclei emerging to the crystal surface with stripe domains is implicated to account for these peculiar domain patterns. Analysis of the spatial correlation functions reveals a particularly slow kinetics, while a crossover to even slower dynamics is found at the late ordering stage. An interaction with intrinsic defects and the consequent effect of random pinning forces causing slow logarithmic growth is inferred to account for the do- main kinetics and dynamical scaling of these deformed configurations. Pattern formation and kinetics at lower temperatures comply with the spatial distribution of domain nuclei observed during switching processes in the submillimeter range for TGS.

\section{ACKNOWLEDGMENTS}

We gratefully acknowledge N. Garcia (CSIC-Madrid) for kindly providing the TGS samples and the EC for financial support within the TMR Network ERBFMRXCT98-0242.
*Present address: Institute of Material Science, NCSR “Demokritos," 15310 Aghia Paraskevi, Athens, Greece.

${ }^{1}$ O. Auciello, J.F. Scott, and R. Ramesh, Phys. Today 52 (7), 22 (1998); T. Hidaka, T. Maruyama, M. Saitoh, N. Mikoshiba, M. Shimizu, T. Shiosaki, L.A. Wills, R. Hiskes, S.A. Diracolis, and J. Amano, Appl. Phys. Lett. 68, 2358 (1996); T. Tybell, C.H. Ahn, and J.-M. Triscone, ibid. 72, 1454 (1998); H. Shin, K.-M. Lee, W.K. Moon, J.U. Jeon, G. Lim, Y.E. Pak, J.H. Park, and K.H. Yoon, IEEE Trans. Ultrason. Ferroelectr. Freq. Control 47, 801 (2000).

${ }^{2}$ A. Gruverman, H. Tokumoto, A.S. Prakash, S. Aggarwal, B. Yang, M. Wuttig, R. Ramesh, O. Auciello, and T. Venkatesan, Appl. Phys. Lett. 71, 3492 (1997); W. Jo, D.C. Kim, and J.W. Hong, ibid. 76, 390 (2000); C.G. Ganpule, V. Nagarajan, S.B. Ogale, A.L. Roytburd, E.D. Williams, and R. Ramesh, ibid. 77, 3275 (2000); A. Gruverman and M. Tanaka, J. Appl. Phys. 89, 1836 (2001).

${ }^{3}$ R. Lüthi, H. Haefke, K.-P. Meyer, E. Meyer, L. Howland, and H.-J. Güntherodt, J. Appl. Phys. 74, 7461 (1993).

${ }^{4}$ F. Saurenbach and B.D. Terris, Appl. Phys. Lett. 56, 1703 (1990).

${ }^{5}$ K. Franke, Ferroelectr. Lett. Sect. 19, 25 (1995); K. Franke and M. Weihnacht, ibid. 19, 35 (1995); K. Franke, H. Huelz, and M. Weihnacht, Surf. Sci. 415, 178 (1998).

${ }^{6}$ G. Zavala, J.H. Fendler, and S.T. McKinstry, J. Appl. Phys. 81, 7480 (1997).

${ }^{7}$ M. Abplanalp, L.M. Eng, and P. Günter, Appl. Phys. A: Mater. Sci. Process. 66, S231 (1998).

${ }^{8}$ L.M. Eng, H.-J. Güntherodt, G. Rosenman, A. Skliar, M. Oron, M. Katz, and D. Eger, J. Appl. Phys. 83, 5973 (1998).

${ }^{9}$ J.W. Hong, K.H. Noh, S. Park, S.I. Kwun, and Z.G. Khim, Phys. Rev. B 58, 5078 (1998).

${ }^{10}$ C. Durkan, M.E. Welland, D.P. Chu, and P. Migliorato, Phys. Rev. B 60, 16198 (1999).

${ }^{11}$ V. Likodimos, X.K. Orlik, L. Pardi, M. Labardi, and M. Allegrini, J. Appl. Phys. 87, 443 (2000).

${ }^{12}$ E.Z. Luo, Z. Xie, J.B. Xu, I.H. Wilson, and L.H. Zhao, Phys. Rev. B 61, 203 (2000).

${ }^{13}$ X.K. Orlik, V. Likodimos, L. Pardi, M. Labardi, and M. Allegrini, Appl. Phys. Lett. 76, 1321 (2000).

${ }^{14}$ V. Likodimos, M. Labardi, and M. Allegrini, Phys. Rev. B 61, 14440 (2000).

${ }^{15}$ V. Likodimos, M. Labardi, X.K. Orlik, L. Pardi, M. Allegrini, S. Emonin, and O. Marti, Phys. Rev. B 63, 064104 (2001).

${ }^{16}$ S.V. Kalinin and D. A Bonnell, Appl. Phys. Lett. 78, 1116 (2001).
${ }^{17}$ S.V. Kalinin and D. A Bonnell, Phys. Rev. B 63, 125411 (2001).

${ }^{18}$ M. Labardi, V. Likodimos, and M. Allegrini, Phys. Rev. B 61, 14390 (2000).

${ }^{19}$ F. Jona and G. Shirane, Ferroelectric Crystals (Pergamon, Oxford, 1962).

${ }^{20}$ M. E. Lines and A. M. Glass, Principles and Applications of Ferroelectrics and Related Materials (Clarendon, Oxford, 1977).

${ }^{21}$ M. Labardi, V. Likodimos, and M. Allegrini, Appl. Phys. A: Mater. Sci. Process. 72, S79 (2001).

${ }^{22}$ J. Hatano, F. Suda, and H. Futama, J. Phys. Soc. Jpn. 43, 1933 (1977).

${ }^{23}$ L. Szczesniak and L. Szczepanska, Ferroelectrics 111, 167 (1990).

${ }^{24}$ K. Takahashi and M. Takagi, J. Phys. Soc. Jpn. 44, 1266 (1978).

${ }^{25}$ N. Nakatani, Ferroelectrics 97, 127 (1989).

${ }^{26}$ K. Hamano, K. Abe, and T. Mitsui, J. Phys. Soc. Jpn. 67, 1037 (1998).

${ }^{27}$ K. Hamano, J. Zhang, K. Abe, T. Mitsui, H. Sakata, and K. Ema, J. Phys. Soc. Jpn. 65, 142 (1996).

${ }^{28}$ A.J. Bray, Adv. Phys. 43, 37 (1994).

${ }^{29}$ B.A. Strukov and A.P. Levanyuk, Ferroelectric Phenomena in Crystals (Springer, Berlin, 1998).

${ }^{30}$ H. Orihara, N. Tomita, and Y. Ishibashi, Ferroelectrics 95, 45 (1989).

${ }^{31}$ N. Tomita, H. Orihara, and Y. Ishibashi, J. Phys. Soc. Jpn. 58, 1190 (1989).

${ }^{32}$ T.J. Yang, V. Gopalan, P.J. Swart, and U. Mohideen, Phys. Rev. Lett. 82, 4106 (1999).

${ }^{33}$ D. Viehland and Y.-H. Chen, J. Appl. Phys. 88, 6696 (2000).

${ }^{34}$ T. Granzow, U. Dörfler, Th. Woike, M. Wöhlecke, R. Pankrath, M. Imlau, and W. Kleemann, Phys. Rev. B 63, 174101 (2001).

${ }^{35}$ D.A. Huse and C.L. Henley, Phys. Rev. Lett. 54, 2708 (1985); D.A. Huse, C.L. Henley, and D.S. Fisher, ibid. 55, 2924 (1985).

${ }^{36}$ T. Nattermann and I. Vilfan, Phys. Rev. Lett. 61, 223 (1988).

${ }^{37}$ T. Nattermann, Y. Shapir, and I. Vilfan, Phys. Rev. B 42, 8577 (1990).

${ }^{38}$ A.J. Bray and K. Humayun, Phys. Rev. E 47, R9 (1993).

${ }^{39}$ S. Puri and N. Parekh, J. Phys. A 25, 4127 (1992); T. Iwai and H. Hayakawa, J. Phys. Soc. Jpn. 62, 1583 (1993); 62, 2971 (1993).

${ }^{40}$ H. Hayakawa, Phys. Rev. B 47, 11696 (1993).

${ }^{41}$ M. Rao and A. Chakrabarti, Phys. Rev. Lett. 71, 3501 (1993).

${ }^{42}$ L.I. Dontsova, N.A. Tikhomirova, and L.A. Shuvalov, Ferroelectrics 97, 87 (1989). 
${ }^{43}$ M.E. Drougard and D.R. Young, Phys. Rev. 94, 1561 (1954).

${ }^{44}$ N.A. Tikhomirova, L.I. Dontsova, A.V. Ginzberg, A.A. Chebotarev, and L.A. Shuvalov, Fiz. Tverd. Tela (Leningrad) 28, 3319 (1986) [Sov. Phys. Solid State 28, 1869 (1986)].
${ }^{45}$ L.I. Dontsova, N.A. Tikhomirova, L.G. Bulatova, E.S. Popov, A.V. Shilnikov, and L.A. Shuvalov, Kristallografiya 28, 388 (1983) [Sov. Phys. Crystallogr. 28, 228 (1983)]. 